\title{
A Diachronic Perspective on the Use of French Spatial Data Infrastructures
}

\section{Jade Georis-Creuseveau ${ }^{1 *}$, Christophe Claramunt ${ }^{2}$, Françoise Gourmelon ${ }^{3}$, Bruno Pinaud ${ }^{4}$, Laurence David ${ }^{3}$}

${ }^{1}$ Laboratory Passages, CNRS, Bordeaux, France

${ }^{2}$ Naval Academy Research Institute, Brest Naval, France

${ }^{3}$ Laboratory LETG-Brest, IUEM, CNRS, Plouzané, France

${ }^{4}$ Laboratory LaBRI, Université de Bordeaux, Bordeaux, France

Email: *jade.georis.creuseveau@gmail.com

How to cite this paper: Georis-Creuseveau, J., Claramunt, C., Gourmelon, F., Pinaud, B. and David, L. (2018) A Diachronic Perspective on the Use of French Spatial Data Infrastructures. Journal of Geographic Information System, 10, 344-361. https://doi.org/10.4236/jgis.2018.104018

Received: June 6, 2018

Accepted: August 5, 2018

Published: August 8, 2018

Copyright (C) 2018 by authors and Scientific Research Publishing Inc. This work is licensed under the Creative Commons Attribution International License (CC BY 4.0).

http://creativecommons.org/licenses/by/4.0/

\begin{abstract}
Despite the recent development of many worldwide initiatives, there is still a need for the development of observation frameworks that will provide a comprehensive view of SDI's use. Amongst the many challenges left, a thorough analysis of the information flows between existing SDIs as well as their respective uses and the way that those evolve over time is an important issue to explore. The research presented in this paper introduces a methodological framework oriented to the study of the SDIs use from a diachronic perspective. The approach is based on a Social Network Analysis (SNA) and questionnaires collected by online surveys. We develop a structural and diachronic analysis based on a series of graph-based measures identifying the main patterns that appear over time. The methodological framework is applied to a series of French SDIs and users involved in environmental management. The study identifies a series of structural differences in the data flows that emerge between the users and SDIs. Last, the diachronic network analysis provides an overall understanding on how data flows evolve over time at different institutional levels.
\end{abstract}

\section{Keywords}

Spatial Data Infrastructure, User, SDIs Use, Social Network Analysis

\section{Introduction}

A Spatial Data Infrastructure (SDIs) can be defined as a dynamic and multi-disciplinary concept that includes technologies, data, policies, institutional arrangements, and people facilitating the availability, understanding, and use of 
spatial data to support decision-making processes within and across applications and scientific domains [1]. Spatial Data Infrastructures cover different levels, varying from Global to local SDIs [2]. They encompass a network of data flows exchanged between organizations that produce, use, and share geographical information [3].

People, stakeholders and organizations are one of SDI key elements for creating, managing, updating and sharing geographical datasets. This can be considered as a collaborative environment in which partnerships are developed across different regional and national borders to promote the exchange of geographical data as exemplified by the Infrastructure for Spatial Information in the European Community (INSPIRE) [4]. INSPIRE is meant to "facilitate the access and use of the various spatial data sources relevant for integrated environmental policy decision making at all governmental levels while supporting the data flow between the local, regional, national and European or international levels" (Mid-term evaluation report on INSPIRE implementation) [5].

Despite the recent development of many worldwide initiatives, it clearly appears that there is still no agreed metrics on how to measure the "use" of such data infrastructures. A key issue still left is the search for "A true measure of use would therefore be the extent to which the use of the infrastructure has achieved to support environmental policies and policies that affect the environment" [5]. However, the search for a comprehensive view of SDI's use, as well as the identification of users "profiles and expectations is far from being a straightforward task" [6] [7] [8] [9]. These challenges are related to the difficulties to acquire a comprehensive view of all the information that flows through these decentralized platforms and to describe these uses and define them in relation to one another. In fact, the use of spatial data amounts at different local and regional scales is largely disseminated and sufficiently flexible to reflect different users' needs.

A major difficulty to assess the usage of SDIs is also due to the heterogeneity dimension of the concept. In fact, the large extent of technological applications and actors involved in the implementation and use of SDI generates a high diversity of application profiles [10]. The relatively recent development of SDIs also generates a high variety of technological, political and administrative implementation contexts [11] [12] [13]. Such heterogeneity is also reflected by the large number of organizations, stakeholders and users involved and the diversity of interactions generated [14] [15]. Last but not least, and in order to observe the impact of SDIs on the long term, the way and to which degree SDIs reflect the evolution of geographical data over time at different levels of local, regional and national scales, as well as the different levels of partners involved should be also taken into account by an observation framework. This is also a difficult task as the integration of time within GIS and moreover SDIs is still a difficult task that deserves the development of sound and conceptual models and data infrastructures [16] [17]. 
In order to develop a methodological framework whose objective will be to evaluate the usability of SDIs, there is clearly a need for the design of an observation and evaluation framework [9]. It has been recently observed that Social Network Analysis (SNA) provides powerful theoretical and computational graph-based methods to analyze the emerging structure and patterns of network-based systems [18]. The SNA combines quantitative and qualitative analysis by the application of computational and visual approaches to outline the main structural properties, identify patterns and contribute to the creation of new insights and knowledge [19]. When applied to the analysis of SDIs, the SNA approach favors an exploration of the structural properties that emerge from the network of interactions between the SDIs and their users [20]. The respective locations of the SDIs and users in the network should give some useful insights on the roles they play in the network, especially when the underlying structure is studied over time.

The research presented in this paper introduces a methodological framework for the study of the SDIs use from a diachronic perspective. The approach focuses on three key patterns of SDI use [3]: SDIs, users and data flows that connect the users to the SDIs. Multiple jurisdictional levels of these flows are taken into account [2] [3], as well as their related dynamics [21]. The aim of the study is to provide a general overview of the evolution of SDIs use based on users, data flows, and institutional levels.

The methodological framework is experimented through a series of French SDIs and users involved in environmental management. This context represents a case study that takes into account a significant number of SDIs implemented at various institutional levels and related to several themes.

The rest of the paper is organized as follows. The next section introduces the main principles of our methodological framework based on time-ordered surveys and Social Network Analysis. Section 3 develops the results of the French case study while Section 4 discusses the main findings and a few perspectives left for further work and Section 5 draws the conclusions and outlines further work.

\section{Methodological Framework}

This section starts with the context of the study and the main principles of the social network analysis applied to a series of data collected in France using online questionnaires conducted in 2012 and 2017, and targeting SDIs and users involved in environment management.

\subsection{Context}

The transposition of Directive 2007/2/CE INSPIRE in France has led to the implementation of a National SDI in the form of a "Géoportail". When compared to other member states in the European Union, France is slightly different as the number of SDIs is relatively large [22].

In 2014, the French association for geographic information (AFIGEO) identified 65 data infrastructures claiming to be SDIs [23]. It appears that the regional 
level alone represents half of the French SDIs identified [23]. They are complemented by thematic (soil, water, coast, etc.) or local (departmental, municipal) infrastructures. This leads us to identify two main SDI's thematic characteristics in France: SDI that provides data to different domains on the one hand, and SDI that provides data to a specific domain (e.g. ocean, forest, soil, etc.) on the other hand [23].

\subsection{Data Collection}

The data collected for the analysis of the SDIs use study originated from two online surveys respectively performed in 2012 and 2017 [9], this giving a five-year distance to allow changes to happen. In both surveys, questions were oriented towards 1) users' institutional context (e.g., type of organization, jurisdictional levels), 2) SDIs used (e.g., type of SDIs, jurisdictional levels), and 3) contribution to decision-making in the environment. The users considered by our study are mostly involved in the implementation of environmental management tasks, and interact with SDIs and data on a more or less regular basis. The two online questionnaires were sent to SDIs users from all over the country. These SDI users were participating on a volunteer basis and responding anonymously.

In 2012, the online survey has generated a database of 446 users who identified 141 SDIs. In 2017, the database has given 256 users who identified 99 SDIs, including 29 common SDIs already mentioned in the survey of 2012. Indeed and as the survey was anonymous, no direct relation can be made between the users that responded in 2012 and 2017 but the two response samples were considered as large enough to provide sufficient patterns to observe.

The SDIs reported by the users are identified thanks to the survey questionnaires. The national and regional SDIs reported by the users are mainly part of the SDIs inventories conducted by the French Association for Geographical Information [23] and by the EUROGI/eSDI-net project [22]. The SDIs which are not part of these inventories are mainly SDIs implemented either after the date of the inventories or at infra-regional or supra-national levels which were not the focus of these inventories.

A deliberative online dissemination strategy of the surveys was applied in order to target a range of users' profiles and SDIs characteristics. Different national professional networks involved in spatial data and environmental management have been asked to distribute broadly the surveys at the two dates. This led to various categories about users' organizations, SDIs thematic characteristics and jurisdictional levels of users and SDIs (Table 1).

\subsection{Data Analysis}

As previously mentioned, this study is based on a SNA whose objective is to investigate the relationships between the SDIs and users based on a graph-based analysis [24]. The relationships between the SDIs and users and the structural 
Table 1. Main characteristics of the users and SDIs that appear from the two online surveys respectively performed in 2012 and 2017.

\begin{tabular}{|c|c|c|}
\hline & $\% 2012$ & $\% 2017$ \\
\hline \multicolumn{3}{|l|}{ Users' organizations } \\
\hline Territorial authority & $39 \%$ & $45 \%$ \\
\hline Private company & $7 \%$ & $7 \%$ \\
\hline Government body & $18 \%$ & $12 \%$ \\
\hline Other (public institution, consular chamber, association...) & $35 \%$ & $35 \%$ \\
\hline \multicolumn{3}{|l|}{ Users jurisdictional levels } \\
\hline Municipal & $42 \%$ & $35 \%$ \\
\hline Departmental & $28 \%$ & $22 \%$ \\
\hline Regional & $21 \%$ & $24 \%$ \\
\hline National & $7 \%$ & $12 \%$ \\
\hline Global & $2 \%$ & $6 \%$ \\
\hline \multicolumn{3}{|l|}{ SDI's thematic characteristics } \\
\hline SDI that provides data to different domains & $64 \%$ & $66 \%$ \\
\hline SDI that provides data to a specific domain (e.g. ocean, forest, soil, etc.) & $36 \%$ & $34 \%$ \\
\hline \multicolumn{3}{|l|}{ SDI's institutional levels } \\
\hline Municipal & $15 \%$ & $3 \%$ \\
\hline Departmental & $16 \%$ & $15 \%$ \\
\hline Regional & $30 \%$ & $42 \%$ \\
\hline National & $20 \%$ & $34 \%$ \\
\hline Global & $20 \%$ & $7 \%$ \\
\hline
\end{tabular}

properties and trends that appear are the main focus of the analysis. The SNA should reveal SDIs use patterns and the related dynamics over time. It explicitly represents the relationships between a series of SDIs and the users interacting with them as part of their professional practices. Moreover, the SNA approach models the network derived from the relationships between the SDIs and the users involved at different institutional levels (i.e., local, regional, national, European). This method is based on a bipartite graph, which is defined as a graph whose nodes can be divided into two distinct sets of entities [25]. The nodes of the bipartite graph are the SDIs and their users. Edges show the relationship between nodes. A SDI is connected by an edge to a user as far as there is an observed data flow between them.

Reflecting the main structural properties of the network involves a range of mathematical measures. Centrality measures are most often used to identify key positions of the nodes within the network while the measure of degree evaluates the interactions of a given SDI or user with respect to its neighborhood (the number of nodes connected to a given node gives its degree [26]. As the graph is bipartite, these measures are computed from all types of nodes (i.e., SDI, user), 
this outlining the respective roles of both SDIs and users at the local and global levels of the emerging network.

In order to observe the overall SDIs changes [27], our study implemented two similar time-ordered networks corresponding to the databases generated by the two online surveys in 2012 and 2017. The network and structural components of the SNA representation have been implemented on top of the SNA software TULIP ${ }^{1}$. TULIP is an information visualization framework dedicated to the analysis and visualization of relational data. Tulip can be used to address a large variety of domain-specific problems using algorithms, visual encodings, interaction techniques, data models, and domain-specific visualizations [28].

\section{Experimental Results}

The SNA approach allows for an exploration of SDIs use based on properties that emerge visually from the networks of interactions between the SDIs and their users at the two given times. As mentioned in the previous section, this graph modeling approach is applied to the answers collected through online surveys conducted in 2012 and 2017 and targeting SDIs users.

\subsection{Towards a SDI Use Combining Multiple SDIs}

Figure 1 (2012) and Figure 2 (2017) show the measure of degree that emerge from the original SNA bipartite graphs and where the number of connections, that is the measures of degree, is given by the size of the spots in the two figures. These two figures outline some typical graph patterns, that is, a series of isolated nodes and peripheral components reflected by some relatively isolated users in 2012 and 2017. Figure 1 and Figure 2 are derived from a packing algorithm implemented in TULIP and that optimizes the visualization of the whole graph. Each graph component is drawn with a force layout algorithm $\left(\mathrm{FM}^{3}\right)$ that converge as much as possible towards a uniform edge length, while the distance between each non-connected pair of nodes is maximized. In particular, this tends to "push" isolated nodes to the external part of the figure, while the most connected nodes are likely to be central.

Figure 1 in 2012 also shows that the most important SDIs in terms of user connections generate isolated clusters, this trend being less important in 2017 as denoted in Figure 2. This also reveals the fact that the number of cross-related connections between different SDIs and users increases progressively over time.

It also appears that the resulting graphs from 2012 and 2017 are highly polarized. A few SDIs are connected with a large number of users, while many SDIs are used by a small number of users. On the user's side, many users use either one SDI or a small numbers of SDIs while a few users are involved in a large number of SDIs.

The measure of degree provides a few additional insights regarding the most connected SDIs and users that appear from the two online surveys respectively 


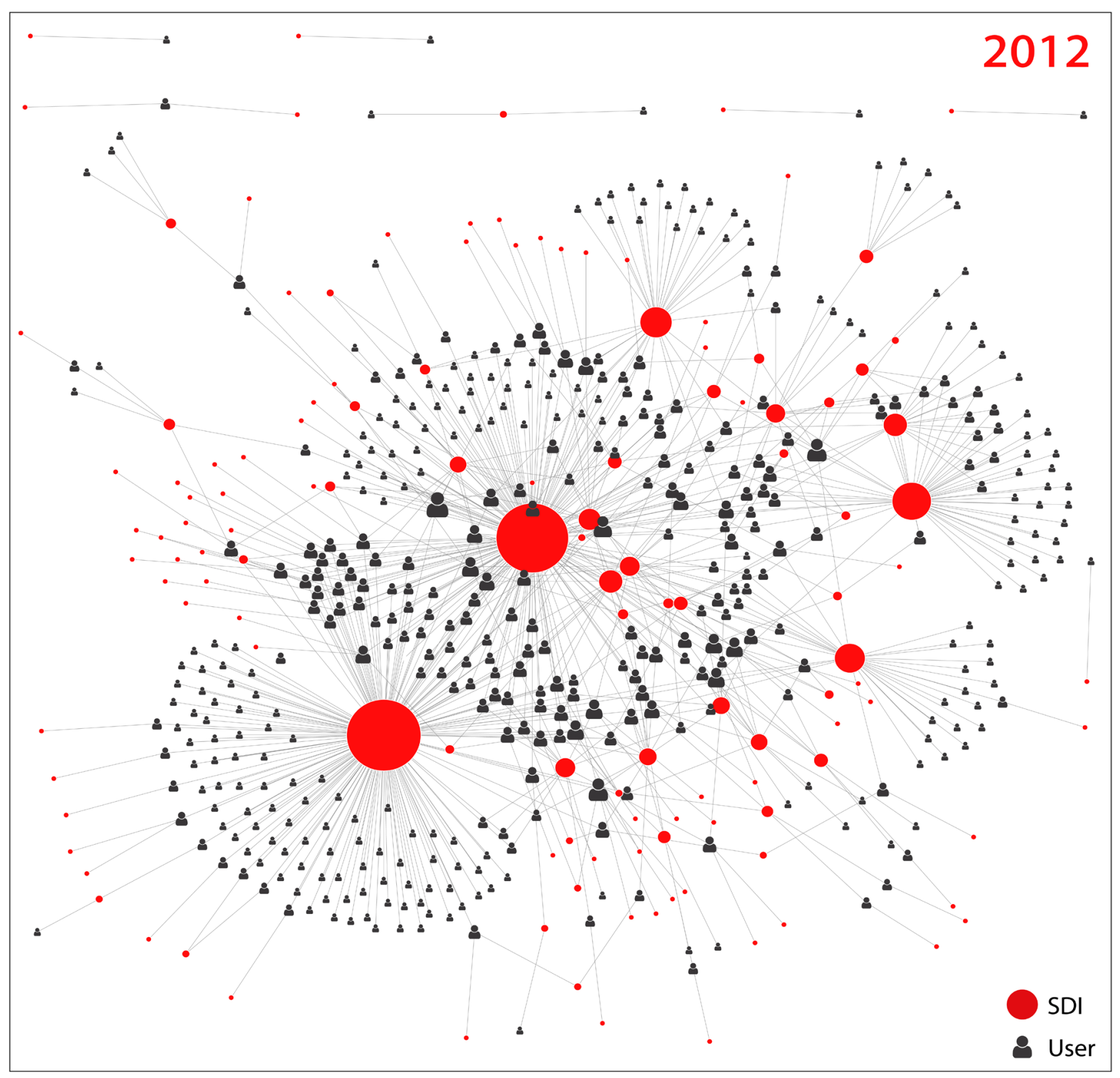

Figure 1. Bipartite graph per degree values-2012.

performed in 2012 and 2017 (see 2.2 data collection). In particular, one can identify the most important SDIs in terms of users' professional practices (Figure 3). In the 2012 graph, the oldest French SDI CRIGE-PACA ${ }^{2}$ is connected to 183 users ( $41 \%$ of the total number of users) while in the 2017 graph, the French national SDI Géoportail ${ }^{3}$ is connected to 126 users (49\% of the total number of users). In 2012, most of the users of CRIGE PACA are only connected to this SDI while in 2017, most of the users of Géoportail are connected to more than one SDI. This denotes not only a decrease in the importance of this SDI, but also the fact that users have a better knowledge of the available SDIs ${ }^{2}$ http://www.geoportail.gouv.fr/accueil ${ }^{3}$ http://www.crige-paca.org/ 


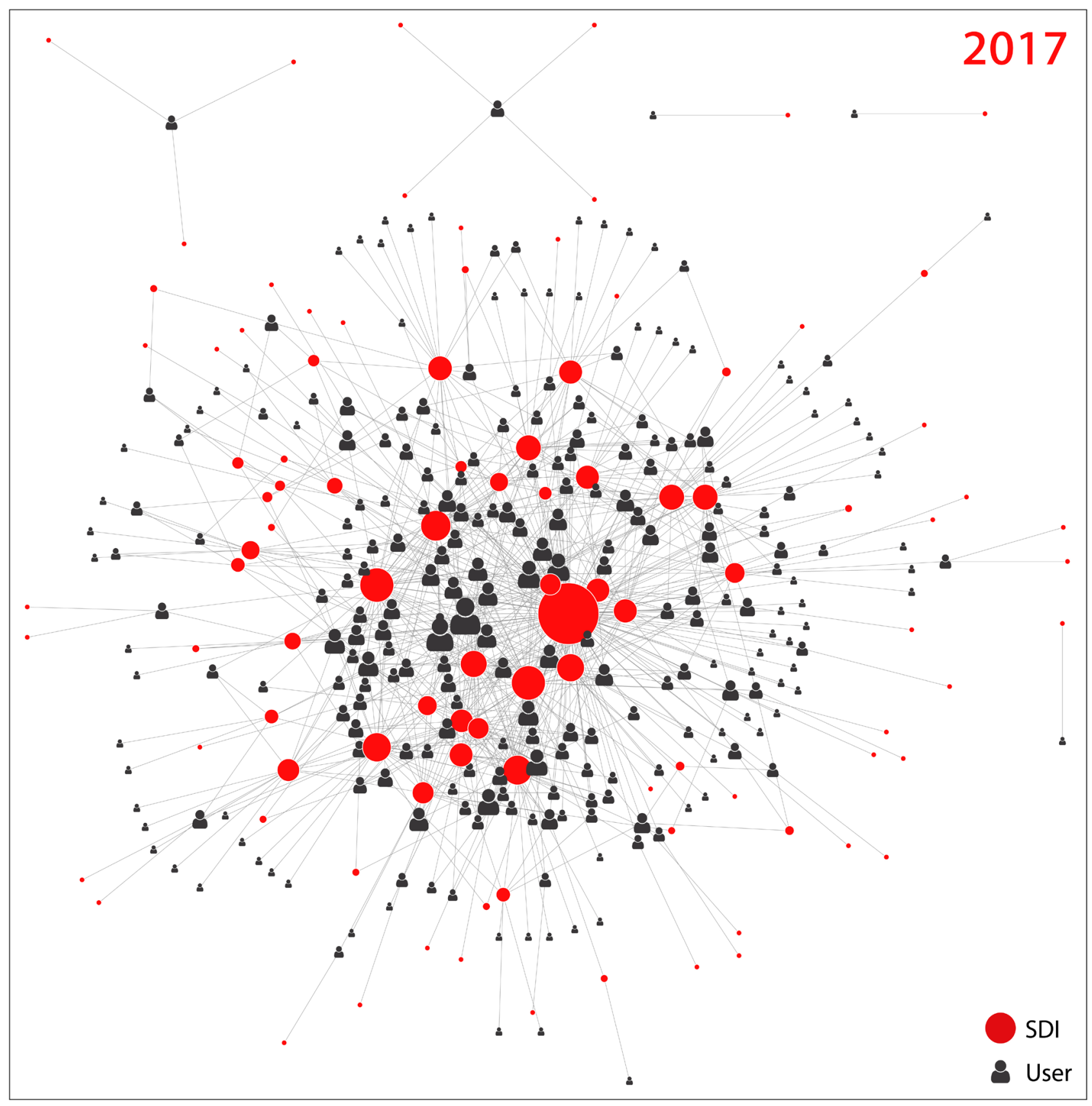

Figure 2. Bipartite graph per degree values-2017.

thus favoring multiple choices and interactions with SDIs at large.

These graphs also reveal some users' practices (Figure 4). For example, in 2012 the most connected user identified $\mathrm{N}^{\circ} 46$ is in interaction with 10 SDIs (accounting for $7 \%$ of the total SDIs in 2012) while in 2017, the most connected user identified $\mathrm{N}^{\circ} 454$ is involved with 20 SDIs (accounting for $20 \%$ of the total SDIs in 2017). Even if a small majority of users (53\% of the total number) combined multiple SDIs in 2012, this trend appears to strengthen in 2017 with almost $70 \%$ of the users ( $69 \%$ of the total number of users) who combine more than one SDI. 

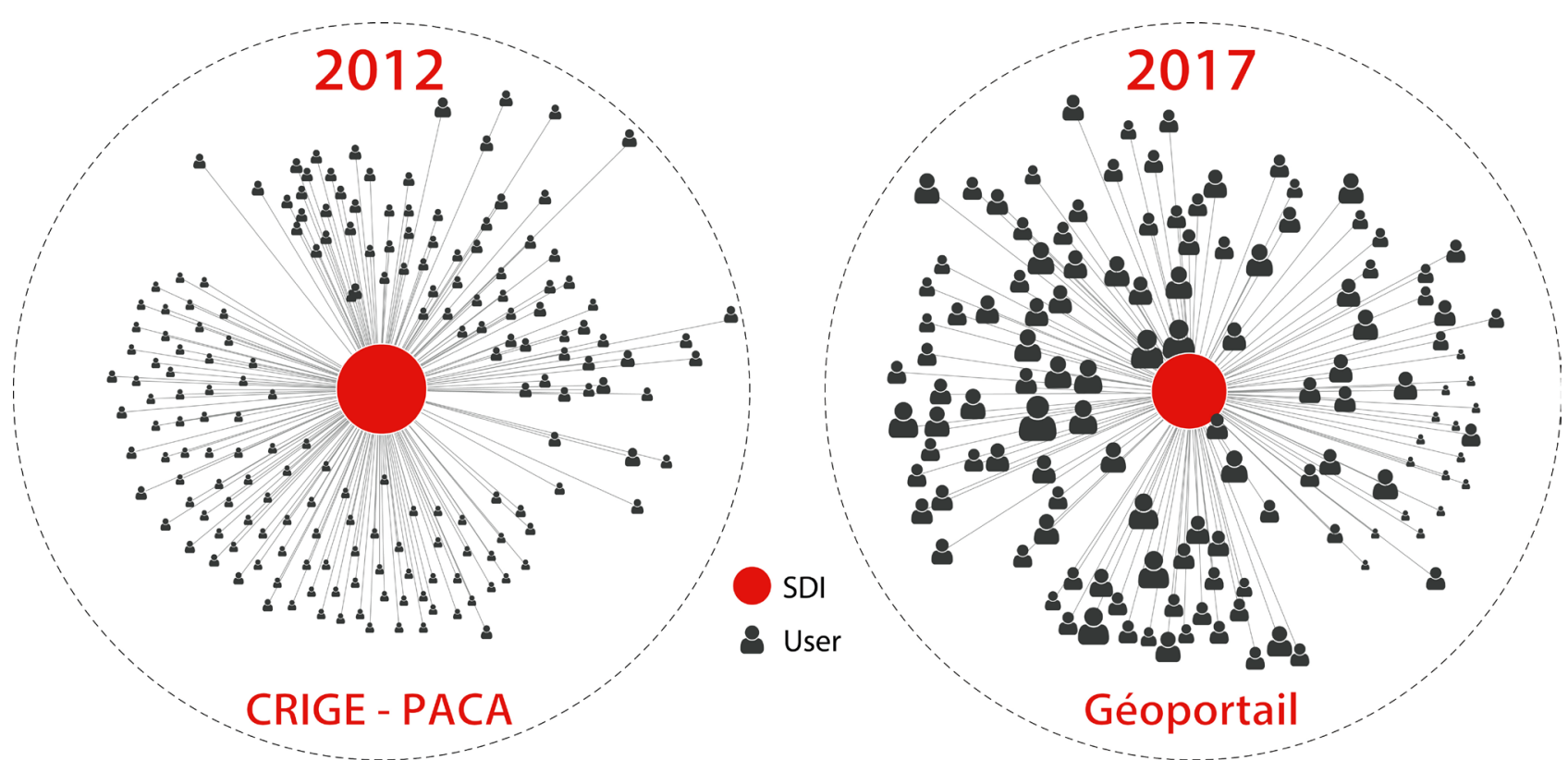

Figure 3. Bipartite graph per degree values—details of the most connected SDIs-2012 \& 2017.

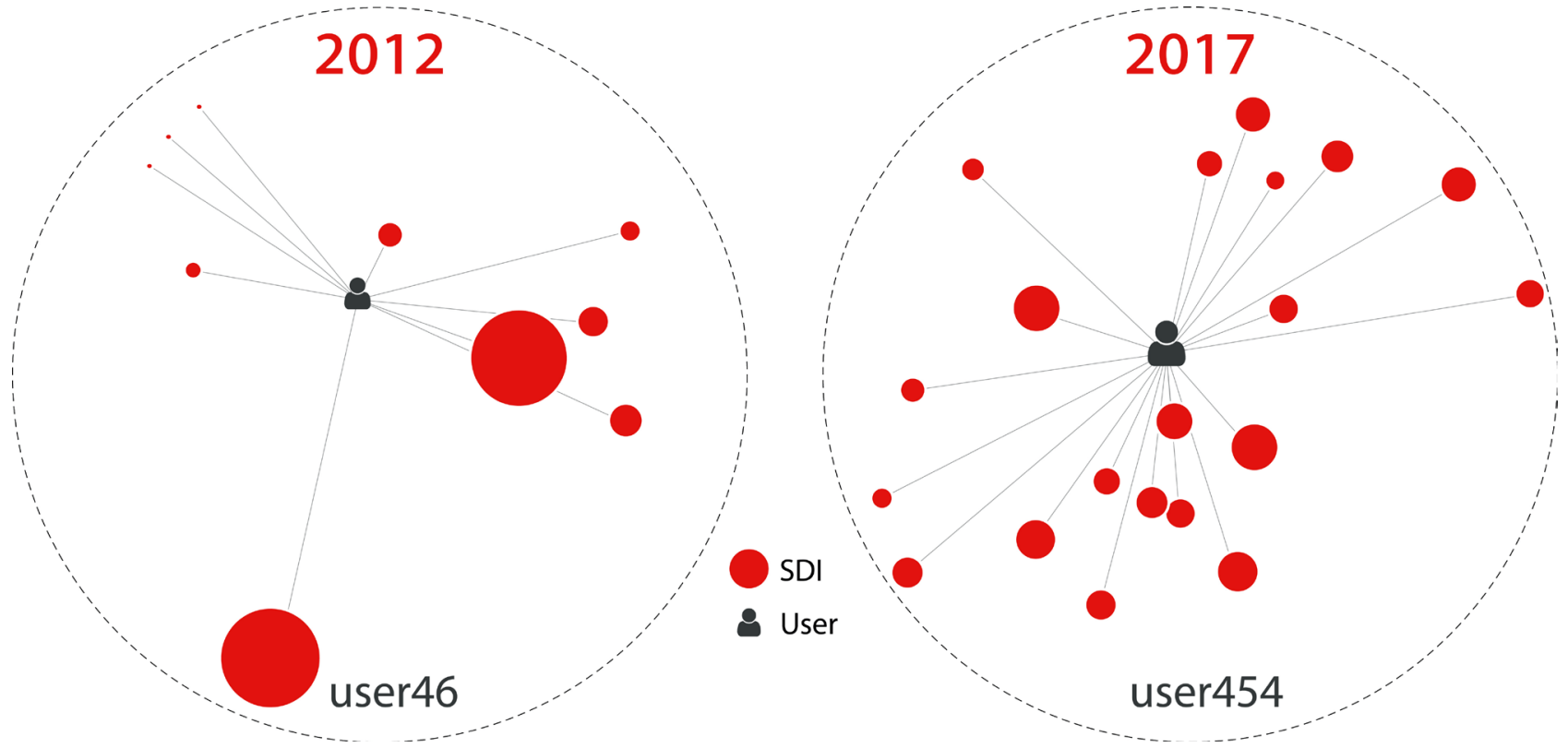

Figure 4. Bipartite graph per degree values-details of the most connected users-2012 \& 2017.

Overall, the SNA analyses reveal that in 2017, most users interact with multiple SDIs. They interact with "key" SDIs (i.e., in the center of the graph) while also interacting with other minor SDIs (i.e., at the periphery).

\subsection{The Multi-Level Dimension of the SDI Use}

Figure 5 (2012) and Figure 6 (2017) explore the way users combine multiple SDIs (called multi-SDIs use). These figures select users involved with more than one SDI, and present a visual hierarchical restructuration of the SNA bipartite graph according to the different institutional levels involved as suggested in our 


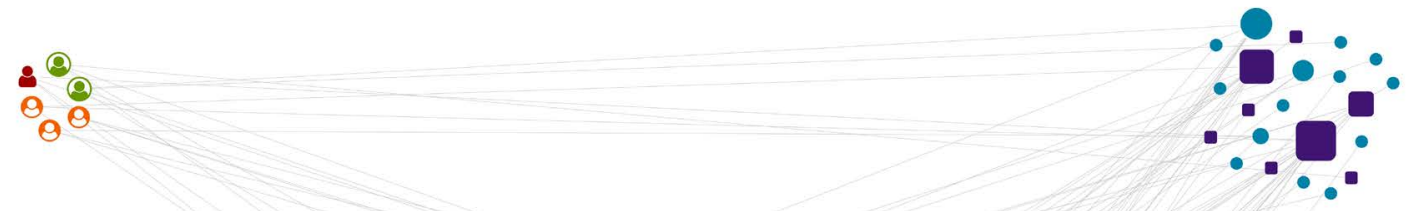

GLOBAL

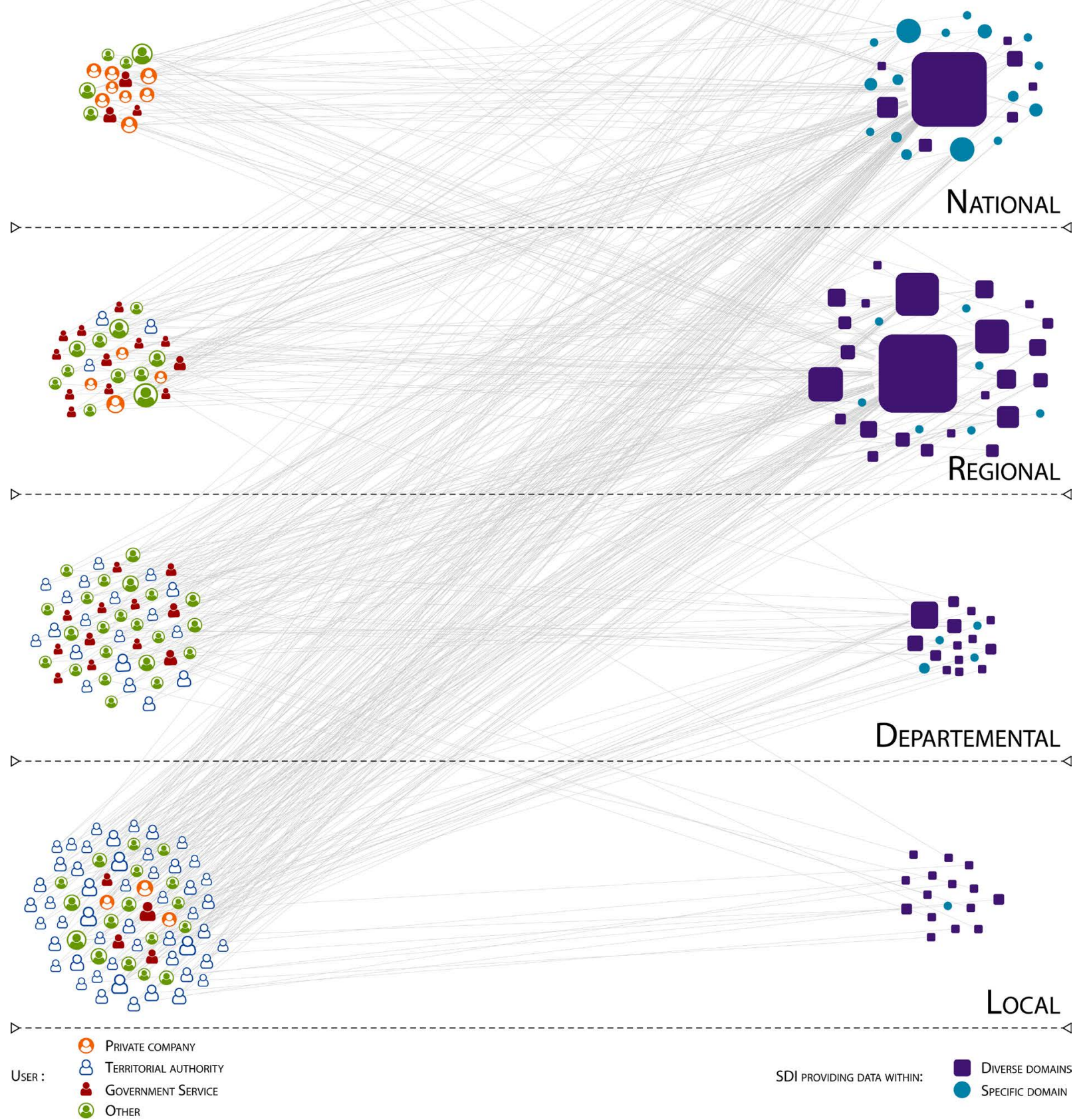

Figure 5. Bipartite graph structured by institutional levels-2012. 
USERS

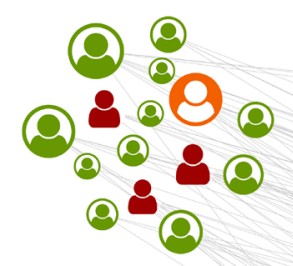

GLOBAL
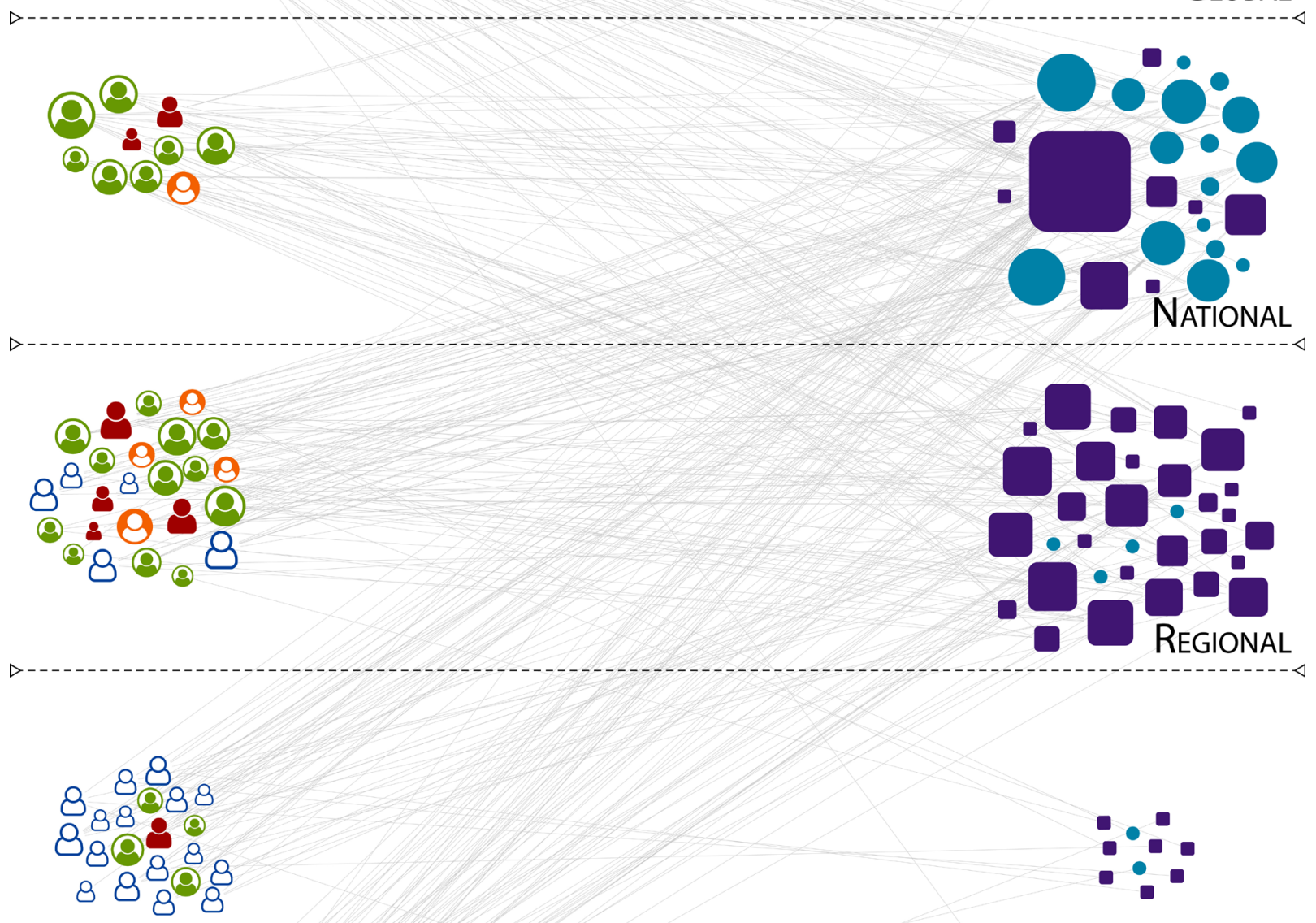

Departemental

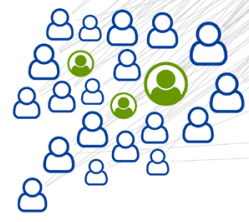

LOCAL

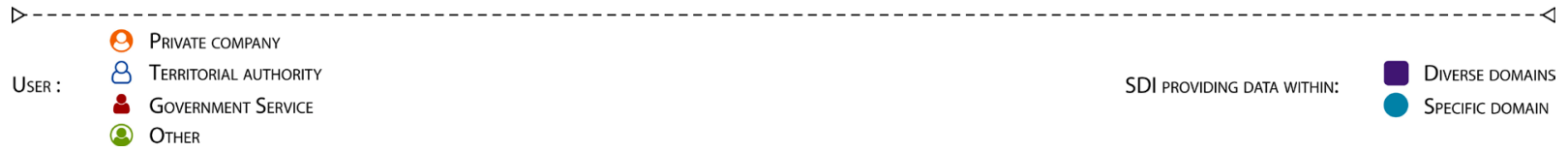

Figure 6. Bipartite graph structured by institutional levels-2017. 
previous work [9]. These institutional levels have been identified from the questionnaire outputs (Table 1). This structural approach takes into account the multi-level dimension of the public policies and by the SDIs implementation in France. Based on the data flows that emerge, this representation outlines the interactions between the different levels and respective role of each level. Network nodes have been also differentiated according to the 4 categories of users' organizations as well as the 4 categories of SDIs characteristics (Table 1). This allows us to illustrate the relative importance of each cluster within the network.

Among the trends that appear, one can remark that combining multiple SDIs is a trend often related to a multi-level use (Figure 5 and Figure 6). Most users interact with SDIs implemented from local to global levels. It also appears that users associated to local or regional institutional levels preferentially use SDIs at the same or higher levels. Users at higher institutional levels (i.e., national and global) are rather characterized by an opposite trend (i.e., identical or lower SDI's levels).

The breakdown of the 4 organizations categories by level are quite similar at the 2 survey dates that allows the comparability between the data collected with the two online surveys respectively performed in 2012 and 2017. Territorial authorities are present from local to regional levels, this being consistent with their spheres of responsibility. Moreover, the distribution of the SDIs characteristics at different levels is comparable at the two survey dates. Albeit implemented at all levels, SDIs that provide data to a specific domain are more represented at the national level at both times.

In 2012 and 2017, the prominent role of national and regional SDIs in France can be clearly observed thanks to the data flows and the measure of degree of the SDIs nodes. However, in 2012, the multi-level representations highlight the important role of regional SDIs. Indeed, regional SDIs are characterized by the greatest number of connections (i.e., 38\% of the graph edges) from the users of all the institutional levels with a preference on the part of municipal users. In 2017, national SDIs now play a central role in shaping the data flows along the network, as more than $42 \%$ of the network edges connect a user to a national SDI. It appears that data flows came from all users' levels with a majority that emerged from the regional level.

The cross-comparison SNA analyses performed between 2012 and 2017 highlights the shift from regional SDIs to national SDIs. This shift can be observed even with similar percentages of SDIs for these two levels at the two dates (Table 1). In 2017, the preference for national SDIs can be observed for the majority of the users, regardless of the nature of the organization to which they belong. For instance, the shift to national SDIs (2017) is particularly observed for the government bodies as $61 \%$ of the network edges connect a user working for government bodies to a national SDI compared to 33\% in 2012 (Figure 7).

We can clearly observe that the majority of the SDIs that provides data for a specific domain are at the national level in 2017, while this being the exception at 


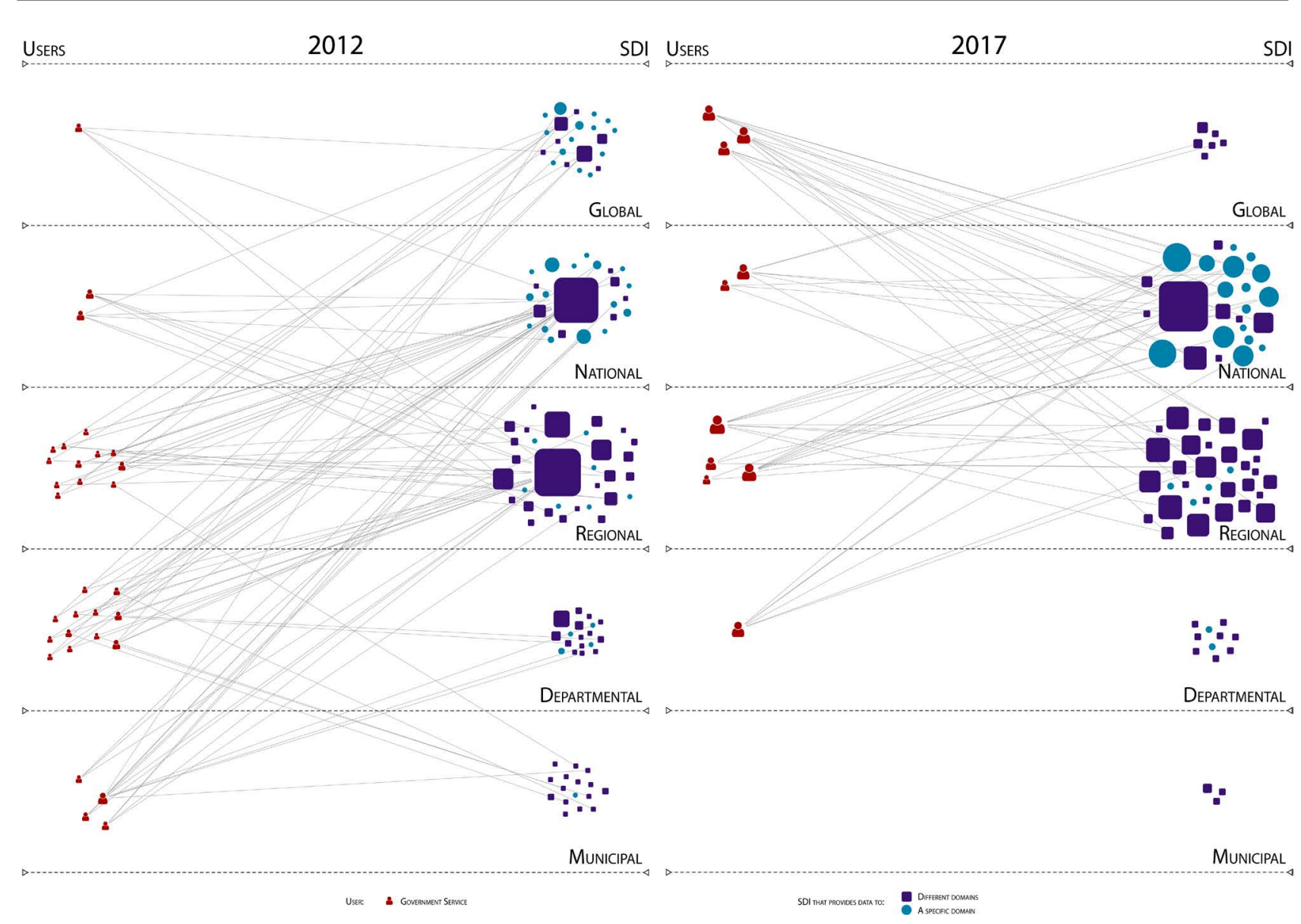

Figure 7. Bipartite graph structured by institutional levels—details of the government bodies—2012 \& 2017 .

all other levels. Moreover, the degree of the SDIs that provides data to a specific domain at the national level is also higher in 2017, this reflecting connections from all users' levels (Figure 8).

\section{Discussion}

The SNA approach developed in this paper illustrates several structural patterns describing the evolution of SDIs use within the French national context. The different patterns and trends that relate the users and SDIs have been identified thanks to several questionnaires collected by online surveys. The study identifies a series of structural differences in the data flows that emerge between the users and SDIs. Furthermore, the diachronic network analysis provides an overall understanding on how data flows evolve over time at different institutional levels. Although the SNA does not provide an assessment on its own, it helps in underpinning several main characteristics of the interactions between the SDIs and the users. This SNA study also outlines the fact that most users combine multiple SDIs, this trend being strengthened over time. The multi-level framework of the SNA highlights the various institutional levels involved in these data flows. The combination of multiple SDIs across various institutional levels reflects the different data levels and scales associated to the users' professional practices. 


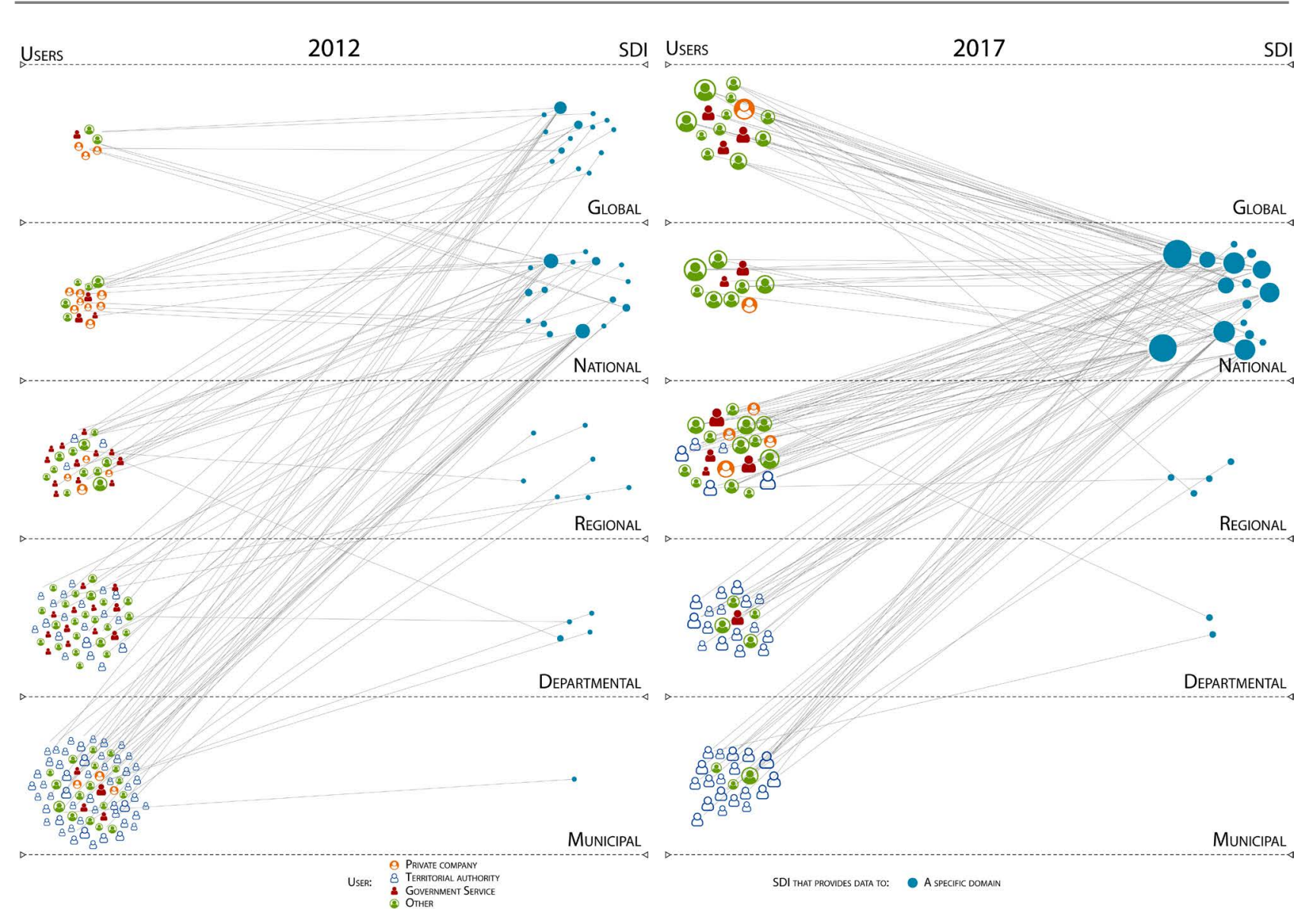

Figure 8. Bipartite graph structured by institutional levels-details of the SDIs that provide data to a specific domain-2012 \& 2017. 
of the EC's INSPIRE to evaluate the progress of its implementation within different member states. We believe that the use itself is a marker of the SDIs' contribution to support environmental policies. Monitoring the main properties and evolutions of SDIs use and data flows should provide some relevant qualitative and quantitative indicators, which, when combined with other dimensions of SDIs (e.g., semantics, data quality, legal issues...) should feed on-going discussions related to the evaluation of SDIs implementation and the INSPIRE Directive (i.e., mid-term evaluation report on INSPIRE implementation 2014).

\section{Conclusions}

This article introduces a methodological approach for the study of the SDIs use based on the interactions between SDIs and their users, as well as the evolution of these interactions. The basic assumption behind this study is that the observation of users, their practices and expectations are key factors for the implementation and development of scalable and flexible SDIs over time [6] [7] [8]. The contribution is twofold. First the approach provides a better understanding of the patterns that appear from an observation of SDI's use at different institutional levels in the specific case of French Spatial Data Infrastructures. Secondly, the contribution is methodological. The approach developed in the paper combines on-line surveys with a Social Network Analysis. The novelty of our study lies in the graph-based analysis that provides a series of quantitative measures and visual capabilities that clearly favour a better understanding of the SDI and user interactions over time.

A direction for further work is to extend the diachronic perspective to additional time frames in order to give a large temporal extent to the whole study and to study the evolution of the whole properties of the SDI infrastructures over a longer period. Another aspect to consider is the spatial dimension of the SDIs use over time. The analysis still deserves to take into account the characteristics and geographical disparities of the uses of the SDIs and their consequence in terms of environment management. This perspective offers the possibility of considering the SDI as geographical objects, through their users, the spatial implications related to their practices and the territorial reorganizations that they are likely to generate. In that context, a worthwhile objective will be to search for the most appropriate method to spatialize the different components of the SDIs, including users. A close integration of the spatial and temporal dimensions should also allow to monitor the geographical distribution of certain types of uses as well as their respective evolution. As such, monitoring the main properties and evolutions of SDIs usage and GI usage should provide some relevant qualitative and quantitative indicators, which, when combined with other dimensions of GI (semantics, data quality, legal issues...) should feed on-going discussions related to Geographic Information Observatories [29].

Another perspective consists of studying why users are interacting with SDIs, complementary to how users use SDIs, the central question of this paper. For 
example, the Unified Theory of Acceptance and Use of Technology [30] could be used for studying the user acceptance processes of SDIs and technologies.

Last but not least this approach is also likely to contribute to a wider research ${ }^{9}$ based on four dimensions: data accessibility, interoperability of information systems, actor networking, and informational equality of territories. The objective here is to consider data and services provided by the SDIs, the users and their practices as well as the strategies of the SDI promoters [20] in order to provide a comprehensive view of institutional spatial data sharing in France.

\section{Acknowledgements}

The authors thank the participants of the online questionnaires conducted in 2012 and 2017.

\section{Conflicts of Interest}

The authors declare no conflicts of interest regarding the publication of this paper.

\section{References}

[1] Rajabifard, A., Feeney, M.-E. and Williamson, I.P. (2002) Future Directions for SDI Development. International Journal of Applied Earth Observation and Geoinformation, 4, 11-22. https://doi.org/10.1016/S0303-2434(02)00002-8

[2] Rajabifard, A., Binns, A., Masser, I. and Williamson, I. (2006) The Role of Sub-National Government and the Private Sector in Future Spatial Data Infrastructures. International Journal of Geographical Information Science, 20, 727-741. https://doi.org/10.1080/13658810500432224

[3] Vandenbroucke, D., Crompvoets, J., Vancauwenberghe, G., Dessers, E. and Orshoven, J. (2009) A Network Perspective on Spatial Data Infrastructures: Application to the Sub-National SDI of Flanders (Belgium). Transactions in GIS, 13, 105-122. https://doi.org/10.1111/j.1467-9671.2009.01166.x

[4] European Commission (2007) Directive 2007/2/EC of the European Parliament and of the Council of 14 March 2007 Establishing an Infrastructure for Spatial Information in the European Community (INSPIRE).

http://eur-lex.europa.eu/LexUriServ/LexUriServ.do?uri=OJ:L:2007:108:0001:0014:E N:PDF

[5] European Environment Agency (2014) Mid-Term Evaluation Report on INSPIRE Implementation. Publications Office of the European Union.

http://www.eea.europa.eu/publications/midterm-evaluation-report-on-inspire-impl ementation

[6] Nedović-Budić, Z., Pinto, J.K. and Budhathoki, N.R. (2008) SDI Effectiveness from the User Perspective. In: Crompvoets, J., et al., Eds., A Multi-View Framework to Assess SDIs, The University of Melbourne, Melbourne, 273-304

[7] Hennig, S., Gryl, I. and Vogler, R. (2013) Spatial Education for Different User Groups as a Prerequisite for Creating a Spatially Enabled Society and Leveraging SDI. International Journal of Spatial Data Infrastructures Research, 8, 98-127

[8] Masser, I. and Crompvoets, J. (2015) Building European Spatial Data Infrastructures.

$\underline{{ }^{\mathrm{http}} / / / \mathrm{www} \text {-iuem.univ-brest.fr/pops/projects/geobs and http://geobs.cnrs.fr/ }}$ 
3rd Edition, ESRI Press, Redlands.

[9] Georis-Creuseveau, J., Claramunt, C. and Gourmelon F. (2017) A Modelling Framework for the Study of Spatial Data Infrastructures Applied to Coastal Management and Planning. International Journal of Geographical Information Science, 37, 122-138. https://doi.org/10.1080/13658816.2016.1188929

[10] Chan, T.O. and Williamson, I.P. (1999) A Model of the Decision Process for GIS Adoption and Diffusion in a Government Environment. Journal of the Urban and Regional Information Systems Association, 11,1-25

[11] Williamson, I.P., Rajabifard, A. and Feeney, M.-E. (2003) Developing Spatial Data Infrastructures: From Concept to Reality. Taylor \& Francis, London. https://doi.org/10.1201/9780203485774

[12] Masser, I. (2005) GIS Worlds: Creating Spatial Data Infrastructures. ESRI Press, Redlands.

[13] Vandenbroucke, D. and Biliouris, D. (2010) Spatial Data Infrastructures in France: State of Play 2011. http://inspire.ec.europa.eu/reports/stateofplay2011/rcr11FRv102.pdf

[14] Grus, L., Crompvoets, J. and Bregt, A. (2010) Spatial Data Infrastructures as Complex Adaptive Systems. International Journal of Geographical Information Science, 24, 439-463. https://doi.org/10.1080/13658810802687319

[15] Barnes, M., Matka, E. and Sullivan, H. (2003) Evidence, Understanding and Complexity: Evaluation in Non-Linear Systems. Evaluation, 9, 265-284.

https://doi.org/10.1177/13563890030093003

[16] Peuquet, D.J. (1994) It's about Time: A Conceptual Framework for the Representation of Temporal Dynamics in Geographic Information Systems. Annals of the Association of American Geographers, 84, 441-461. https://doi.org/10.1111/j.1467-8306.1994.tb01869.x

[17] Thériault, M., Claramunt, C., Séguin, A.M. and Villeneuve, P. (2002) Temporal GIS and Statistical Modelling of Personal Lifelines. In: Richardson, D.E. and van Oosterom, P., Eds., Advances in Spatial Data Handling, Springer, Berlin, Heidelberg, 433-449. https://doi.org/10.1007/978-3-642-56094-1_32

[18] Otte, E. and Rousseau, R. (2002) Social Network Analysis: A Powerful Strategy, Also for the Information Science. Journal of Information Science, 28, 441-453. https://doi.org/10.1177/016555150202800601

[19] Brandes, U., Kenis, P. and Raab, J. (2006) Explanation through Network Visualization. Methodology, 2, 16-23. https://doi.org/10.1027/1614-2241.2.1.16

[20] Noucher, M., Gourmelon, F., Gautreau, P., Georis-Creuseveau, J., Maulpoix, A., Pierson, J., Pinède, N., Pissoat, O. and Rouan, M. (2017) Spatial Data Sharing: A Pilot Study of French SDIs. ISPRS International Journal of Geo-Information, 6, 99. https://doi.org/10.3390/ijgi6040099

[21] Harvey, F., Iwaniak, A., Coetzee, S. and Cooper, A.K. (2012) SDI Past, Present and Future: A Review and Status Assessment. GSDI, Quebec, 23.

[22] EUROGI (2012) SDI Best Practice Database. http://www.esdinetplus.eu/best_practice/database.html

[23] AFIGEO (2014) Catalogue des IDG 2014. Association Française pour l'Information Geographique. http://afigeo.asso.fr/voir-toutes-les-news/972-3-juin-2014-la-nouvelle-version-du-c atalogue-des-idg.html

[24] Wasserman, S. and Faust, K. (1994) Social Network Analysis: Methods and Applica- 
tions. Cambridge University Press, Cambridge. https://doi.org/10.1017/CBO9780511815478

[25] Borgatti, S.P. and Everett, M.G. (1997) Network Analysis of 2-Mode Data. Social Networks, 19, 243-269. https://doi.org/10.1016/S0378-8733(96)00301-2

[26] Hanneman, R.A. and Riddle, M. (2005) Introduction to Social Network Methods. University of California, Riverside, $114 \mathrm{p}$. http://faculty.ucr.edu/ hanneman/nettext/

[27] Knoke, D. and Yang, S. (2008) Social Network Analysis. SAGE, Thousand Oaks. https://doi.org/10.4135/9781412985864

[28] Auber, D., Archambault, D., Bourqui, R., Delest, M., Dubois, J., Lambert, A., Mary, P., Mathiaut, M., Mélançon, G. and Pinaud, B. (2017) TULIP 5. Encyclopedia of Social Network Analysis and Mining, 1-28. https://doi.org/10.1007/978-1-4614-7163-9_315-1

[29] Janowicz, K., Adams, B., McKenzie, G. and Kauppinen, T. (2014) Towards Geographic Information Observatories. 11 th Conference on Spatial Information Theory, Scarborough, 2014, 100-1006.

[30] Venkatesh, V., Morris, M.G., Davis, G.B. and David, F.D. (2003) User Acceptance of Information Technology: Toward a Unified View. MIS Quarterly, 27, 425-478. https://doi.org/10.2307/30036540 